\title{
Effects of chronic L-theanine administration in patients with major depressive disorder: an open-label study
}

Hidese S, Ota M, Wakabayashi C, Noda T, Ozawa H, Okubo T, Kunugi H. Effects of chronic L-theanine administration in patients with major depressive disorder: an open-label study.

Objective: L-theanine, an amino acid uniquely contained in green tea (Camellia sinensis), has been suggested to have various psychotropic effects. This study aimed to examine whether L-theanine is effective for patients with major depressive disorder (MDD) in an open-label clinical trial. Methods: Subjects were 20 patients with MDD (four males; mean age: $41.0 \pm 14.1$ years, 16 females; $42.9 \pm 12.0$ years $)$. L-theanine $(250 \mathrm{mg} /$ day $)$ was added to the current medication of each participant for 8 weeks. Symptoms and cognitive functions were assessed at baseline, 4 , and 8 weeks after L-theanine administration by the 21 -item version of the Hamilton Depression Rating Scale (HAMD-21), State-Trait Anxiety Inventory (STAI), Pittsburgh Sleep Quality Index (PSQI), Stroop test, and Brief Assessment of Cognition in Schizophrenia (BACS).

Results: HAMD-21 score was reduced after L-theanine administration $(p=0.007)$. This reduction was observed in unremitted patients (HAMD-21 $>7 ; p=0.004$ ) at baseline. Anxiety-trait scores decreased after L-theanine administration $(p=0.012)$ in the STAI test. PSQI scores also decreased after L-theanine administration $(p=0.030)$ in the unremitted patients at baseline. Regarding cognitive functions, response latency $(p=0.001)$ and error rate $(p=0.036)$ decreased in the Stroop test, and verbal memory $(p=0.005)$ and executive function $(p=0.016)$ were enhanced in the BACS test after L-theanine administration.

Conclusion: Our study suggests that chronic (8-week) L-theanine administration is safe and has multiple beneficial effects on depressive symptoms, anxiety, sleep disturbance and cognitive impairments in patients with MDD. However, since this is an open-label study, placebo-controlled studies are required to consolidate the effects.

\author{
Shinsuke Hidese ${ }^{1,2}$, \\ Miho Ota ${ }^{1}$, Chisato \\ Wakabayashi', \\ Takamasa Noda ${ }^{3}$, \\ Hayato Ozawa ${ }^{4}$, Tsutomu \\ Okubo4, Hiroshi Kunugi, \\ ${ }^{1}$ Department of Mental Disorder Research, \\ National Institute of Neuroscience, National \\ Center of Neurology and Psychiatry, Tokyo, \\ Japan; ${ }^{2}$ Department of NCNP Brain Physiology \\ and Pathology, Division of Cognitive and \\ Behavioral Medicine, Graduate School of \\ Medical and Dental Sciences, Tokyo Medical \\ and Dental University, Tokyo, Japan; \\ ${ }^{3}$ Department of Psychiatry, National Center \\ Hospital, National Center of Neurology and \\ Psychiatry, Tokyo, Japan; and ${ }^{4}$ Department of \\ Research and Development, Nutrition Division, \\ Taiyo Kagaku Co. Ltd, Mie, Japan
}

Keywords: anxiety; cognitive function; depression; L-theanine; sleep

Dr. Hiroshi Kunugi, Department of Mental Disorder Research, National Institute of Neuroscience, National Center of Neurology and Psychiatry, 4-1-1 Ogawa-Higashi, Kodaira, Tokyo 187-8502, Japan

Tel: +81 42346 1714;

Fax: +81 42346 2094;

E-mail: hkunugi@ncnp.go.jp

Accepted for publication June 9, 2016

First published online 11 July 2016

\section{Significant outcomes}

- Depressive symptoms, anxiety, and sleep disturbance were improved after L-theanine administration in patients with major depressive disorder (MDD).

- Cognitive functions were enhanced after L-theanine administration in patients with MDD.

- High tolerability of L-theanine was suggested.

\section{Limitations}

- This study was an open-label trial.

- The majority of the subjects was affected by relatively mild forms of MDD.

- The number of the subjects was small. 


\section{Introduction}

MDD is a serious mental illness presenting with depressed mood, anxiety, sleep disturbance, various somatic symptoms, and cognitive impairments $(1,2)$. Although many antidepressants acting on synaptic monoamine levels have been used as the first-line drug treatment for MDD, they are not effective in a substantial proportion of patients (3). In addition, they can often elicit intolerable side effects in patients. This justifies the development of new treatment strategies in terms of efficacy and tolerability for patients suffering from MDD (4). Several natural compounds (e.g. St. John's wort, omega-3 fatty acids, and $S$-adenosyl-L-methionine) have been reported to be effective for MDD, whereas having fewer side effects and relatively low placeboresponse rates than antidepressants $(5,6)$.

L-theanine ( $\mathrm{L}-\gamma$-glutamylethylamide) is a nonproteinous amino acid, which is uniquely contained in green tea (Camellia sinensis). L-theanine crosses the blood-brain barrier, and is transported into the brain in a dose-dependent manner (7). The chemical structure of L-theanine resembles that of L-glutamate and competes with it on glutamate receptors (8). In experiments on rats, stress-induced suppression of recognition memory was rescued by L-theanine administration, which recovered the attenuation of in vitro hippocampal CA1 long-term potentiation (LTP) after stress exposure (9). Recognition memory was also enhanced in the L-theanine-administered rats, showing increased population spike amplitude in the induction of in vivo hippocampal dentate gyrus LTP (10). It has been shown that L-theanine has various psychotropic effects (11). In experiments on mice, depression-like behaviour and cognitive dysfunction in a confront-housing condition, a model of chronic psychosocial stress, were suppressed in the L-theanine intake group (12). We also reported the antidepressant-like effect of L-theanine together with the agonistic action on $\mathrm{N}$-methyl-D-aspartate receptors (13). It has also been shown that anxietyassociated behaviours decrease (14), and that chronic stress-induced cognitive impairments improve (15) in the L-theanine-administered mice.

In healthy humans, L-theanine was effective in reducing state anxiety scores, as well as in decreasing sympathetic nerve responses following acute stress task (16). In electroencephalogram studies, L-theanine affected both resting state (17) and attention taskrelated $(18,19) \quad \alpha$-wave activity which influences prefrontal cortical function, contributing to cognitive processes associated with attention and memory (20). Error rates in the attention task decreased in healthy participants (21), and cognitive functions, including attention and memory, also improved in subjects exhibiting mild cognitive impairment (22) in the L-theanine-administered group. Further, L-theanine showed augmentative effects on antipsychotic therapy in patients with schizophrenia and schizoaffective disorder, improving positive, activation, dysphoric mood, and anxiety symptoms (23). We have also reported similar symptom-reducing effects of L-theanine in patients with schizophrenia (24).

\section{Aims of the study}

Although L-theanine has been shown to have various psychotropic effects, there has been no clinical study performed to show whether this compound affects symptoms and cognitive functions in patients with MDD. The aim of this study was to examine the psychotropic effects of L-theanine in patients with MDD.

\section{Material and methods}

Subjects

Subjects (mean age: $42.6 \pm 12.1$ years, four males and 16 females) were 20 patients with the diagnosis of MDD according to the criteria in the Diagnostic and Statistical Manual of Mental Disorders, 5th edition (25). Each diagnosis was based on information obtained by the Mini-International Neuropsychiatric Interview (26), and additional unstructured interviews, and medical records where available. We included some remitted patients with MDD as well as unremitted patients at baseline in order to elucidate the possible effects of L-theanine on mild forms of the illness. Participants were excluded if they had prior medical histories of central nervous system disease, severe head injury, substance abuse, or mental retardation. All patients were recruited from the outpatients of the National Center of Neurology and Psychiatry Hospital $(n=6)$ or from the local community through prepared advertisements $(n=14)$. After describing the study, written informed consent was obtained from every subject. Thereafter, patient anonymity has been preserved. Before starting the study, we registered the protocol at the university hospital medical information network - clinical trials registry and complied with it accordingly (UMIN000014653).

\section{L-theanine administration}

After the initial psychiatric assessments, L-theanine tablets $(250 \mathrm{mg} /$ day) (Suntheanine, Taiyo Kagaku Co. Ltd, Japan) were orally administered before sleep for 8 weeks. This dose was chosen because it was effective in our prior study (24). Psychiatric 
symptoms were assessed at baseline, 4 and 8 weeks after L-theanine administration. In total, 12 patients $(60 \%)$ were treated with antidepressant medication at baseline and this medication was unchanged during our intervention period. Daily doses of antidepressants were converted to imipramine-equivalent doses using a published Japanese guideline (27). The mean imipramine-equivalent dose was calculated in the total subjects $(n=20)$. Participants were instructed not to alter their usual intakes of green tea during the intervention period.

\section{Clinical assessments}

To evaluate depressive symptoms, anxiety, and sleep state, the 21-item version of the GRID Hamilton Depression Rating Scale (HAMD-21) (28,29), State-Trait Anxiety Inventory (STAI) (30), and Pittsburgh Sleep Quality Index (PSQI) (31) were used. Adverse events were assessed using the Udvalg for Klinicke Unders $\emptyset$ gelser (UKU) side effect rating scale (32). Cognitive functions were measured by the Stroop test (33) and the Brief Assessment of Cognition in Schizophrenia (BACS) (34). The Stroop test was conducted on a computer using two types (control and experimental condition) of PowerPoint files composed of four (red, blue, yellow, and black) coloured words (35). After the participants practiced by the control condition in which colour and word were consistent, response latency (ms) and error rate (\%) were evaluated in the experimental condition in which colour and word were inconsistent. Two versions (A and B) of BACS were used to exclude any learning effects.

\section{Laboratory tests}

Non-fasting blood samples were obtained before clinical assessments. Serum samples were prepared by centrifuging bloods for $10 \mathrm{~min}$ at 3,000 rotations/ min. Plasma samples were prepared to measure haemoglobin A1c. The samples were evaluated at baseline, 4 and 8 weeks after L-theanine administration at SRL Co. Ltd, Japan to check general physical states and monitor side effects. Serum L-theanine blood concentrations were measured by a highperformance liquid chromatography system based on the AccQ.Tag method as described in the literature (36) using commercially available reagents and equipment (Waters Corp., Tokyo, Japan).

\section{Statistics}

Differences in clinical assessments and laboratory data between baseline and 8 weeks after L-theanine administration were analysed by the Wilcoxon's signed-rank test. Effect sizes were calculated using $r$ values. All statistical tests were two-tailed and $p<0.05$ indicated statistical significance. Statistical analyses were performed using the Statistical Package for the Social Sciences version 22.0 (IBM Corp., Tokyo, Japan).

\section{Results}

The clinical characteristics of the subjects are summarised in Table 1. All participants completed the entire 8-week study, suggesting high tolerability of L-theanine. Time courses of symptom scores are shown in Table 2. Six HAMD-21 subscale scores were calculated as described in Seretti et al. (37). There was a significant decrease in HAMD-21 total scores $(p=0.007, r=-0.61)$, and a significant reduction in four subscale scores (core, psychic anxiety, somatic anxiety, and delusion) and a tendency of reduction in sleep subscale scores in the total patients $(n=20)$. When the patients were divided into unremitted patients (HAMD-21 $>7 ; n=13$ ) at baseline and the remaining remitted patients $(n=7)$ according to Riedel et al. (38), the former showed a significant decrease $(p=0.004, r=-0.79)$, whereas the latter did not $(p=0.35, r=-0.36)$ (Fig. 1). Among the unremitted patients at baseline, six $(46.2 \%)$ were responders $(50 \%$ or more reduction of HAMD-21 score) and six (46.2\%) became remitters at 8 weeks. Among the remitted patients at baseline, there were only two (28.6\%) responders. We did not detect any significant association of clinical variables (age, sex, height, weight, body mass index, education, onset of illness, single/recurrent episode, imipramineequivalent dose, or smoking) with response to L-theanine (data not shown).

STAI consisted of anxiety-state and anxiety-trait indices. We found a tendency of reduction in anxietystate $(p=0.058, r=-0.43)$, and a significant reduction in anxiety-trait $(p=0.012, r=-0.56)$

Table 1. The clinical characteristics of the subjects

\begin{tabular}{lcc}
\hline & Mean \pm SD & Range \\
\hline Age (years) & $42.6 \pm 12.1$ & $20-67$ \\
Sex [male: $n(\%)]$ & $4(20)$ & \\
Height $(\mathrm{cm})$ & $161.7 \pm 7.3$ & $151-178$ \\
Weight $(\mathrm{kg})$ & $62.0 \pm 16.3$ & $43-96$ \\
Body mass index $\left(\mathrm{kg} / \mathrm{m}^{2}\right)$ & $23.5 \pm 4.8$ & $17-33$ \\
Education (years) & $15.8 \pm 4.0$ & $12-26$ \\
Onset of illness (years) & $30.9 \pm 14.4$ & $13-67$ \\
Single/recurrent episode $[n(\%)]$ & $9 / 11(45 / 55)$ & \\
Imipramine-equivalent dose $(\mathrm{mg} /$ day) & $101.7 \pm 131.8$ & $0-525$ \\
Smoking $[n(\%)]$ & $2(10)$ & \\
HAMD-21 score at baseline & $12.5 \pm 8.0$ & $4-33$ \\
\hline
\end{tabular}

HAMD-21, 21-item version of the Hamilton Depression Rating Scale. 
Table 2. Time courses of symptom scores after L-theanine administration

\begin{tabular}{|c|c|c|c|c|}
\hline & Baseline (mean \pm SD) & 4 weeks (mean \pm SD) & 8 weeks (mean \pm SD) & Wilcoxon's signed-rank test \\
\hline HAMD-21 & $12.5 \pm 8.0$ & $8.5 \pm 6.3$ & $8.8 \pm 7.8$ & $W=19.5,{ }^{* *} p=0.007, r=-0.61$ \\
\hline Non-remitters* & $16.2 \pm 7.6$ & $10.6 \pm 6.7$ & $10.7 \pm 8.1$ & $W=1.0,{ }^{* *} p=0.004, r=-0.79$ \\
\hline Remitters $^{\dagger}$ & $5.7 \pm 1.4$ & $4.4 \pm 2.5$ & $5.3 \pm 6.4$ & $W=6.0, p=0.35, r=-0.36$ \\
\hline Core & $6.9 \pm 3.4$ & $4.5 \pm 3.4$ & $5.1 \pm 4.4$ & $W=31.5,{ }^{*} p=0.018, r=-0.53$ \\
\hline Sleep & $1.0 \pm 1.2$ & $0.7 \pm 1.1$ & $0.6 \pm 0.8$ & $W=8.0, p=0.070, r=-0.41$ \\
\hline Activity & $1.7 \pm 1.3$ & $0.8 \pm 1.1$ & $1.3 \pm 1.5$ & $W=29.0, p=0.12, r=-0.42$ \\
\hline Psychic anxiety & $1.7 \pm 1.1$ & $1.0 \pm 0.8$ & $1.1 \pm 0.9$ & $W=11.0,{ }^{*} p=0.012, r=-0.57$ \\
\hline Somatic anxiety & $2.5 \pm 2.1$ & $1.8 \pm 1.4$ & $1.7 \pm 1.7$ & $W=8.0,{ }^{*} p=0.023, r=-0.51$ \\
\hline Delusion & $1.9 \pm 1.6$ & $1.2 \pm 1.5$ & $1.0 \pm 1.3$ & $W=17.1,{ }^{*} p=0.024, r=-0.51$ \\
\hline \multicolumn{5}{|r|}{ (e } \\
\hline Anxiety-state & $52.9 \pm 11.5$ & $51.5 \pm 11.2$ & $49.5 \pm 11.6$ & $W=48.0, p=0.058, r=-0.43$ \\
\hline Non-remitters* & $56.6 \pm 11.5$ & $54.1 \pm 12.4$ & $52.5 \pm 12.2$ & $W=25.5, p=0.16, r=-0.39$ \\
\hline Remitters $^{\dagger}$ & $45.9 \pm 8.0$ & $46.6 \pm 7.0$ & $43.9 \pm 8.5$ & $W=2.0, p=0.072, r=-0.68$ \\
\hline Anxiety-trait & $58.2 \pm 13.0$ & $54.3 \pm 11.8$ & $53.5 \pm 12.8$ & $W=33.0,{ }^{*} p=0.012, r=-0.56$ \\
\hline Non-remitters* & $61.8 \pm 13.6$ & $57.1 \pm 12.0$ & $56.0 \pm 13.6$ & $W=18.0, p=0.054, r=-0.53$ \\
\hline Remitters $^{\dagger}$ & $51.6 \pm 9.1$ & $49.1 \pm 10.5$ & $48.7 \pm 10.4$ & $W=1.0,{ }^{*} p=0.046, r=-0.76$ \\
\hline PSOl & $9.6 \pm 3.7$ & $8.8 \pm 3.2$ & $9.1 \pm 2.6$ & $W=41.5, p=0.16, r=-0.31$ \\
\hline Non-remitters* & $10.6 \pm 3.8$ & $9.5 \pm 3.6$ & $9.5 \pm 2.9$ & $W=6.5,{ }^{*} p=0.030, r=-0.60$ \\
\hline Remitters $^{\dagger}$ & $7.6 \pm 2.8$ & $7.4 \pm 1.8$ & $8.1 \pm 1.8$ & $W=12.5, p=0.67, r=0.16$ \\
\hline
\end{tabular}

HAMD-21, 21-item version of the Hamilton Depression Rating Scale; PSOI, Pittsburgh Sleep Quality Index; STAI, State-Trait Anxiety Inventory; W, Wilcoxon's signed-rank test statistic.

* HAMD-21>7 $(n=13)$

${ }^{\dagger} \mathrm{HAMD}-21 \leq 7(n=7)$.

${ }^{*} p<0.05,{ }^{* *} p<0.01$.

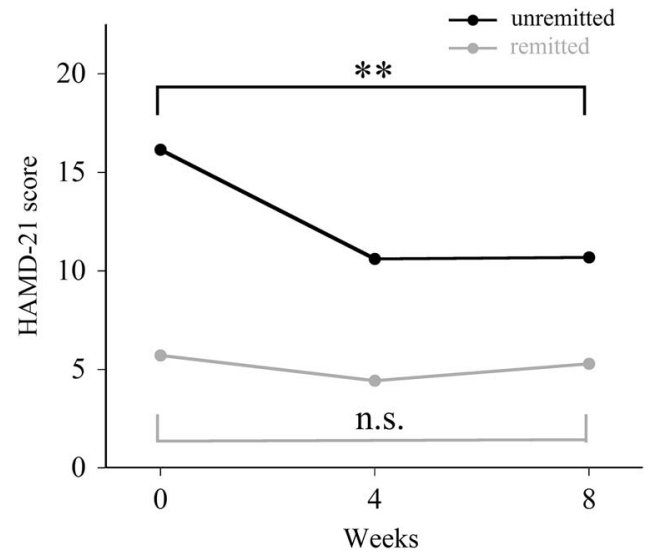

Fig. 1. Twenty-one-item version of the Hamilton Depression Rating Scale (HAMD-21) score change after L-theanine administration. There was a significant reduction of HAMD-21 scores in unremitted patients $(n=13)$, whereas such a significant change was not observed in remitted patients ( $n=7$, grey coloured). n.s., Not significant. $* * p<0.01$.

after L-theanine administration in the total patients. PSQI scores were significantly reduced after L-theanine administration in the patients who were unremitted at baseline $(p=0.030, r=-0.60)$. We found no notable adverse events in the UKU side effect rating scale (4 weeks: $0.2 \pm 0.5,8$ weeks: $0.3 \pm 0.6)$. One patient reported slight sleepiness, two patients reported increased duration of sleep of up to
$2 \mathrm{~h}$ longer than usual, and two patients reported slightly increased dream activity.

Time courses of cognitive function scores are shown in Table 3. In the Stroop test, response latency was significantly shortened after L-theanine administration ( $p=0.001, r=-0.72)$. There was also a significant decrease in error rate in the Stroop test $(p=0.036, r=-0.47)$. Among seven indices of BACS, verbal memory score $(p=0.005, r=0.63)$ and executive function score $(p=0.016, r=0.54)$ were significantly enhanced after L-theanine administration.

Changes in laboratory data are shown in Table 4. Except for a significant decrease of high-density lipoprotein (HDL) cholesterol $(p=0.011, r=-0.57$ ), general metrics such as liver function, renal function, and glucose metabolism were not significantly altered after L-theanine administration. As expected, L-theanine blood concentration significantly increased after administration ( $p=0.005, r=0.64)$.

\section{Discussion}

We examined the possible effects of L-theanine in an open-label clinical trial. We found that psychiatric symptoms and cognitive functions of patients with MDD were improved after L-theanine administration. HAMD-21 scores, an objective index of depression, were reduced in unremitted patients at baseline; 
Hidese et al.

Table 3. Time courses of cognitive function scores after L-theanine administration

\begin{tabular}{|c|c|c|c|c|}
\hline & Baseline $($ mean $\pm S D)$ & 4 weeks $($ mean $\pm S D)$ & 8 weeks (mean \pm SD) & Wilcoxon's signed-rank test \\
\hline \multicolumn{5}{|l|}{ Stroop test } \\
\hline Response latency (ms) & $1216 \pm 324$ & $1130 \pm 286$ & $1080 \pm 307$ & $W=18.5,{ }^{* *} p=0.001, r=-0.72$ \\
\hline Error rate $(\%)$ & $2.4 \pm 2.3$ & $1.5 \pm 1.5$ & $1.2 \pm 1.4$ & $W=13.0,{ }^{*} p=0.036, r=-0.47$ \\
\hline \multicolumn{5}{|l|}{ BACS } \\
\hline Verbal memory & $48.1 \pm 9.7$ & $48.7 \pm 10.5$ & $53.2 \pm 8.0$ & $W=164.5,{ }^{* *} p=0.005, r=0.63$ \\
\hline Working memory & $22.0 \pm 3.2$ & $21.6 \pm 3.1$ & $21.5 \pm 3.3$ & $W=66.0, p=0.61, r=-0.11$ \\
\hline Motor speed & $79.0 \pm 15.3$ & $83.2 \pm 14.6$ & $81.8 \pm 17.4$ & $W=121.0, p=0.29, r=0.24$ \\
\hline Category fluency & $22.9 \pm 6.6$ & $24.9 \pm 7.6$ & $24.6 \pm 6.1$ & $W=101.5, p=0.24, r=0.27$ \\
\hline Letter fluency & $27.8 \pm 8.7$ & $28.4 \pm 8.7$ & $29.8 \pm 10.1$ & $W=143.5, p=0.15, r=0.32$ \\
\hline Attention & $64.0 \pm 13.5$ & $63.2 \pm 12.4$ & $66.1 \pm 14.6$ & $W=121.5, p=0.12, r=0.35$ \\
\hline Executive function & $17.5 \pm 2.4$ & $17.2 \pm 2.6$ & $19.0 \pm 1.7$ & $W=102.0,{ }^{*} p=0.016, r=0.54$ \\
\hline
\end{tabular}

BACS, Brief Assessment of Cognition in Schizophrenia; W, Wilcoxon's signed-rank test statistic.

${ }^{*} p<0.05,{ }^{* *} p<0.01$.

Table 4. Changes in laboratory data after L-theanine administration

\begin{tabular}{|c|c|c|c|c|}
\hline & Baseline $($ mean \pm SD) & 4 weeks (mean $\pm S D$ ) & 8 weeks (mean \pm SD) & Wilcoxon's signed-rank test \\
\hline AST (U/I) & $23.8 \pm 14.0$ & $21.5 \pm 9.7$ & $23.3 \pm 11.9$ & $W=59.5, p=0.42, r=-0.18$ \\
\hline ALT (U/I) & $22.9 \pm 19.7$ & $22.3 \pm 18.6$ & $21.5 \pm 16.9$ & $W=80.0, p=0.81, r=-0.05$ \\
\hline$\gamma$-GTP $(\mathrm{U} / \mathrm{I})$ & $30.0 \pm 30.0$ & $27.8 \pm 31.2$ & $32.0 \pm 35.9$ & $W=106.5, p=0.36, r=0.21$ \\
\hline CRE (mg/dl) & $0.7 \pm 0.1$ & $0.7 \pm 0.1$ & $0.7 \pm 0.1$ & $W=70.0, p=0.50, r=-0.15$ \\
\hline $\mathrm{BUN}(\mathrm{mg} / \mathrm{dl})$ & $12.6 \pm 3.7$ & $12.4 \pm 3.8$ & $11.9 \pm 3.3$ & $W=79.0, p=0.33, r=-0.22$ \\
\hline $\mathrm{T}-\mathrm{CHO}$ (mg/dl) & $197.3 \pm 27.5$ & $192.2 \pm 32.1$ & $192.0 \pm 30.5$ & $W=77.0, p=0.30, r=-0.23$ \\
\hline $\mathrm{HDL}-\mathrm{CHO}(\mathrm{mg} / \mathrm{dl})$ & $62.0 \pm 16.5$ & $60.0 \pm 15.2$ & $57.6 \pm 13.7$ & $W=27.0,{ }^{*} p=0.011, r=-0.57$ \\
\hline LDL-CHO (mg/dl) & $110.0 \pm 18.9$ & $105.4 \pm 28.0$ & $107.3 \pm 24.6$ & $W=86.0, p=0.48, r=-0.16$ \\
\hline $\mathrm{TG}(\mathrm{mg} / \mathrm{dl})$ & $126.6 \pm 96.1$ & $127.9 \pm 96.6$ & $135.3 \pm 93.0$ & $W=141.5, p=0.17, r=0.31$ \\
\hline $\mathrm{Hb}$ A1c (\%) & $5.8 \pm 1.3$ & $5.7 \pm 1.2$ & $5.8 \pm 1.4$ & $W=107.0, p=0.63, r=0.11$ \\
\hline
\end{tabular}

ALT, alanine transaminase; AST, aspartate transaminase; BUN, blood urea nitrogen; CRE, creatinin; GTP, glutamyltranspeptidase; Hb, haemoglobin; HDL-CHO, high-density lipoprotein cholesterol; LDL-CHO, low-density lipoprotein cholesterol; T-CHO, total cholesterol; TG, triglyceride; $W$, Wilcoxon's signed-rank test statistic. ${ }^{*} p<0.05$.

although the score-reducing effect was not observed any more in patients who were already remitted at baseline. Among the HAMD-21 subscales, core, psychic anxiety, somatic anxiety, and delusion scores were reduced. The scores in STAI and PSQI metrics were also reduced. Response latency and error rate decreased in the Stroop test and verbal memory and executive function increased in the BACS. Furthermore, there were no dropout subjects and no serious adverse events in either UKU side effect rating scale or laboratory data. To our knowledge, we showed, for the first time, reduced symptoms and enhanced cognitive functions in patients with MDD after L-theanine administration, suggesting that it possesses multiple favourable psychotropic effects.

Depressive symptoms were significantly reduced after L-theanine administration, which suggests that it has antidepressant effects. This is in line with previous reports in mice $(12,13)$. Considering the structural commonality of L-theanine to L-glutamate, the primary site of action is likely to be the glutamatergic pathway which is a therapeutic target for depression $(39,40)$. Meta-analyses have reported that ketamine, a non-competitive glutamate receptor antagonist, is effective in treating depressive disorders $(41,42)$. L-theanine has a lower affinity for glutamate receptors than does L-glutamate itself (8). We showed that L-theanine could exert a partially agonistic effect on glutamate receptors (13) and regulate the concentration of glutamate and glutamine in the brain (24). Although detailed mechanisms are unknown, it is possible that, similar to a low dose of ketamine (43), L-theanine might normalise impaired glutamatergic transmission in MDD.

We found a tendency for reduction in anxiety-state scores and a significant reduction in anxiety-trait scores after L-theanine administration. This may suggest its anxiolytic effects in our patients with MDD, which is corroborated by previous studies in mice and humans $(14,16,23)$. This effect might be attributable again to the action of L-theanine on glutamate receptors, because modulators of the 
receptors have shown their anxiolytic effects (44). Increased level of central nervous system inhibitory neurotransmitters, such as glycine (45) and $\gamma$-aminobutyric acid (46), following theanine administration may also contribute to the anxiolytic effects. Further, we found a significant improvement of sleep disturbance after L-theanine administration in our patients with MDD. In line with this, L-theanine has been suggested to help ameliorate sleep quality in patients with MDD as in attention deficit hyperactivity disorder (47) and schizophrenia (24).

We found enhanced cognitive functions after L-theanine administration in line with previous reports in rats, mice, and humans $(9,10,12,15,21,22)$. Although one recent double-blind randomised controlled trial reported that L-theanine had no beneficial effect on cognition in healthy coffee drinkers (48), the dose (50 mg) was substantially lower than ours $(250 \mathrm{mg})$. Further, it was single, but not chronic L-theanine administration. In the Stroop test, response latency and error rates were significantly reduced in patients with MDD. The Stroop task is relevant to prefrontal cortical activity and other conflict-processing functional areas in the brain (49). In the BACS, verbal memory and executive function scores of the patients significantly improved in this study. L-theanine may improve verbal memory and executive function by modulating the involved cortical areas through its putative $\alpha$-wave activity related to attention and memory in healthy subjects in electroencephalogram studies (17-19).

As discussed in Godsil et al. (50), the hippocampus-prefrontal pathway might be related with the treatment response to L-theanine administration. In the hippocampus and prefrontal cortex, glutamatergic signalling is an essential regulator underlying synaptic plasticity. Disrupted function in this system can constitute the pathology of MDD and present a novel therapeutic target or research of antidepressant (51). Considering that stress-related impairment of hippocampal synaptic plasticity was recovered (9), and that the augmentation of hippocampal synaptic plasticity was observed (10) in L-theanine-administered rats, the antidepressant and cognition-enhancing effects might also be related to the effect on synaptic plasticity.

In laboratory data, HDL cholesterol, alternatively called as 'good' cholesterol, was significantly reduced after L-theanine administration. This result is opposite to a report that the levels of HDL cholesterol increased in the theanine-supplemented group in hepatoma-bearing rats (52). Although the mechanisms underlying our result were elusive, there were no subjects who newly showed the value below the lower limit of reference range $(40 \mathrm{mg} / \mathrm{dl})$ at 8 weeks.
There are several limitations in our study. First, we cannot exclude the influence of the placebo effect on our results. Placebo-controlled clinical trials are required to confirm the therapeutic effect of L-theanine as an adjunct therapy in the treatment of MDD. Second, although a significant reduction in depressive symptoms were seen after L-theanine administration, the mean HAMD-21 score at baseline was 12.5 points, indicating that the patients presented relatively mild forms of MDD, which might have obscured the possible antidepressant effect.

In conclusion, reduced depressive symptoms, anxiety, sleep disturbance, and enhanced cognitive functions were found in patients with MDD after chronic (8-week) L-theanine $(250 \mathrm{mg} /$ day) administration. We also found high tolerability of L-theanine. These results suggest that L-theanine could be a useful natural compound in the treatment of MDD.

\section{Acknowledgements}

The authors thank Ikki Ishida, Moeko Hiraishi, Anna Nagashima, and Dr. Junko Matsuo for their assistance in the recruitment of subjects. Authors' Contributions: S.H., M.O. and H.K. designed the study and H.K. supervised the results. C.W. was involved in supporting the intellectual content. T.N. helped recruitment of subjects from the outpatient clinic. T.O. provided important knowledge about L-theanine and critical comments to the study. H.O. carried out highperformance liquid chromatography analysis. S.H. evaluated clinical assessments, executed statistical analyses, and wrote the manuscript, which was revised and approved by all authors.

\section{Financial Support}

This study was supported by an Intramural Research Grant (M.O. and H.K., grant number 24-11, 27-1) for Neurological and Psychiatric Disorders of National Center of Neurology and Psychiatry, and an unrestricted research grant provided by the Taiyo Kagaku Co. Ltd.

\section{Conflicts of Interest}

H.O. and T.O. are employees of Taiyo Kagaku Co. Ltd., which provided L-theanine tablets used in the present study, and various food products.

\section{Ethical Standards}

The authors assert that all procedures contributing to this work comply with the ethical standards of the ethics committee of National Center of Neurology and Psychiatry and with the Helsinki Declaration of 1975, as revised in 2008. 
Hidese et al.

\section{References}

1. KenNedy SH. Core symptoms of major depressive disorder: relevance to diagnosis and treatment. Dialogues Clin Neurosci 2008;10:271-277.

2. Rock PL, Roiser JP, Riedel WJ, Blackwell AD. Cognitive impairment in depression: a systematic review and metaanalysis. Psychol Med 2014;44:2029-2040.

3. HAMON M \& BLIER P. Monoamine neurocircuitry in depression and strategies for new treatments. Progress in NeuroPsychopharmacology and Biological Psychiatry 2013;45:54-63.

4. Rush AJ, Trivedi MH, Wisniewski SR et al. Acute and longer-term outcomes in depressed outpatients requiring one or several treatment steps: a STAR*D report. Am J Psychiatry 2006;163:1905-1917.

5. Freeman MP, Mischoulon D, Tedeschini E et al. Complementary and alternative medicine for major depressive disorder: a meta-analysis of patient characteristics, placebo-response rates, and treatment outcomes relative to standard antidepressants. J Clin Psychiatry 2010;71:682-688.

6. NAHAS R, Sheikh O. Complementary and alternative medicine for the treatment of major depressive disorder. Can Fam Physician 2011;57:659-663.

7. Yokogoshi H, Kobayashi M, Mochizuki M, Terashima T. Effect of theanine, r-glutamylethylamide, on brain monoamines and striatal dopamine release in conscious rats. Neurochem Res 1998;23:667-673.

8. Kakuda T, Nozawa A, Sugimoto A, Nino H. Inhibition by theanine of binding of $[3 \mathrm{H}] \mathrm{AMPA},[3 \mathrm{H}]$ kainate, and $[3 \mathrm{H}]$ MDL 105,519 to glutamate receptors. Biosci Biotechnol Biochem 2002;66:2683-2686.

9. Tamano H, Fukura K, Suzuki M, Sakamoto K, Yokogoshi H, TAKEDA A. Preventive effect of theanine intake on stressinduced impairments of hippocamapal long-term potentiation and recognition memory. Brain Res Bull 2013;95:1-6.

10. Tamano H, Fukura K, Suzuki M, Sakamoto K, Yokogoshi H, TAKEDA A. Advantageous effect of theanine intake on cognition. Nutr Neurosci 2014;17:279-283.

11. LARDNER AL. Neurobiological effects of the green tea constituent theanine and its potential role in the treatment of psychiatric and neurodegenerative disorders. Nutr Neurosci 2014;17:145-155.

12. Unno K, Fumitani K, Takamori $\mathrm{N}$ et al. Theanine intake improves the shortened lifespan, cognitive dysfunction and behavioural depression that are induced by chronic psychosocial stress in mice. Free Radic Res 2011;45: 966-974.

13. Wakabayashi C, Numakawa T, Ninomiya M, Chiba S, Kunugi H. Behavioral and molecular evidence for psychotropic effects in L-theanine. Psychopharmacology 2012;219:1099-1109.

14. Wise LE, Premaratne ID, Gamage TF et al. L-theanine attenuates abstinence signs in morphine-dependent rhesus monkeys and elicits anxiolytic-like activity in mice. Pharmacol Biochem Behav 2012;103:245-252.

15. Tian X, Sun L, Gou L et al. Protective effect of L-theanine on chronic restraint stress-induced cognitive impairments in mice. Brain Res 2013;1503:24-32.

16. Kimura K, Ozeki M, Juneja LR, Ohira H. L-Theanine reduces psychological and physiological stress responses. Biol Psychol 2007;74:39-45.

17. Nobre AC, RaO A, Owen GN. L-theanine, a natural constituent in tea, and its effect on mental state. Asia Pac J Clin Nutr 2008;17(Suppl. 1):167-168.
18. Gomez-Ramirez M, Kelly SP, Montesi JL, Foxe JJ. The effects of L-theanine on alpha-band oscillatory brain activity during a visuo-spatial attention task. Brain Topogr 2009;22:44-51.

19. Gomez-Ramirez M, Higgins BA, Rycroft JA et al. The deployment of intersensory selective attention: a highdensity electrical mapping study of the effects of theanine. Clin Neuropharmacol 2007;30:25-38.

20. Knyazev GG. Motivation, emotion, and their inhibitory control mirrored in brain oscillations. Neurosci Biobehav Rev 2007;31:377-395.

21. Foxe JJ, Morie KP, Laud PJ, Rowson MJ, de Bruin EA, Kelly SP. Assessing the effects of caffeine and theanine on the maintenance of vigilance during a sustained attention task. Neuropharmacology 2012;62:2320-2327.

22. Park SK, Jung IC, LeE WK et al. A combination of green tea extract and L-theanine improves memory and attention in subjects with mild cognitive impairment: a double-blind placebo-controlled study. J Med Food 2011;14:334-343.

23. Ritsner MS, Miodownik C, RAtner $Y$ et al. L-theanine relieves positive, activation, and anxiety symptoms in patients with schizophrenia and schizoaffective disorder: an 8-week, randomized, double-blind, placebo-controlled, 2-center study. J Clin Psychiatry 2011;72:34-42.

24. Ота M, Wakabayashi C, Sato $\mathrm{N}$ et al. Effect of L-theanine on glutamatergic function in patients with schizophrenia. Acta Neuropsychiatr 2015;27:291-296.

25. American Psychiatric Association. Diagnostic and Statistical Manual of Mental Disorders, 4th edn. Arlington, VA: American Psychiatric Association, 2013.

26. Sheehan DV, Lecrubier Y, Sheehan KH et al. The MiniInternational Neuropsychiatric Interview (M.I.N.I.): the development and validation of a structured diagnostic psychiatric interview for DSM-IV and ICD-10. J Clin Psychiatry 1998;59(Suppl. 20):22-33.

27. InAda T, InAGaki A. Psychotropic dose equivalence in Japan. Psychiatry Clin Neurosci 2015;69:440-447.

28. Williams JB, Kовaк KA, BeCh P et al. The GRID-HAMD: standardization of the Hamilton Depression Rating Scale. Int Clin Psychopharmacol 2008;23:120-129.

29. Hamilton M. A rating scale for depression. J Neurol Neurosurg Psychiatry 1960;23:56-62.

30. Spielberg CD. GRLaLRE. STAI Manual. Palo Alto, CA: Consulting Psychologists Press, 1970.

31. Buysse DJ, Reynolds CF 3Rd, Monk TH, Berman SR, KUPFER DJ. The Pittsburgh Sleep Quality Index: a new instrument for psychiatric practice and research. Psychiatry Res 1989;28:193-213.

32. Linguaerde O, Ahlfors UG, Bech P, Dencker SJ, Elgen K. The UKU side effect rating scale. A new comprehensive rating scale for psychotropic drugs and a cross-sectional study of side effects in neuroleptic-treated patients. Acta Psychiatr Scand Suppl 1987;334:1-100.

33. Stroop JR. Studies of interference in serial verbal reactions. J Exp Psychol 1935;18:643-662.

34. Keefe RS, Goldberg Te, Harvey PD, Gold JM, Poe MP, Coughenour L. The Brief Assessment of Cognition in Schizophrenia: reliability, sensitivity, and comparison with a standard neurocognitive battery. Schizophr Res 2004;68: 283-297.

35. MACLEOD CM, MACDonald PA. Interdimensional interference in the Stroop effect: uncovering the cognitive and neural anatomy of attention. Trends Cogn Sci 2000;4:383-391. 
36. Gardener MC, Gillman MP. Analyzing variability in nectar amino acids: composition is less variable than concentration. J Chem Ecol 2001;27:2545-2558.

37. Seretti A, Cusin C, Lattuada E, Di Bella D, Catalano M, SMERALDi E. Serotonin transporter gene (5-HTTLPR) is not associated with depressive symptomatology in mood disorders. Mol Psychiatry 1999;4:280-283.

38. Riedel M, Moller HJ, Obermeier M et al. Response and remission criteria in major depression - a validation of current practice. J Psychiatr Res 2010;44:1063-1068.

39. SKolnick P, PopiK P, Trullas R. Glutamate-based antidepressants: 20 years on. Trends Pharmacol Sci 2009;30:563-569.

40. НАлнімото K. Emerging role of glutamate in the pathophysiology of major depressive disorder. Brain Res Rev 2009;61:105-123.

41. Newport DJ, Carpenter LL, McDonald WM, Potash JB, Tohen M, Nemeroff CB, Biomarkers APACoRTFoN, \& Treatments. Ketamine and other NMDA antagonists: early clinical trials and possible mechanisms in depression. Am J Psychiatry 2015;172:950-966.

42. Caddy C, Giaroli G, White TP, Shergill SS, Tracy DK. Ketamine as the prototype glutamatergic antidepressant: pharmacodynamic actions, and a systematic review and meta-analysis of efficacy. Ther Adv Psychopharmacol 2014;4:75-99.

43. Miller OH, Moran JT, Hall BJ. Two cellular hypotheses explaining the initiation of ketamine's antidepressant actions: direct inhibition and disinhibition. Neuropharmacology 2016;100:17-26.

44. Bergink V, van Megen HJ, Westenberg HG. Glutamate and anxiety. Eur Neuropsychopharmacol 2004;14:175-183.
45. Yamada T, Terashima T, Okubo T, Juneja LR, Yokogoshi H. Effects of theanine, r-glutamylethylamide, on neurotransmitter release and its relationship with glutamic acid neurotransmission. Nutr Neurosci 2005;8:219-226.

46. Kimura R, Murata T. Influence of alkylamides of glutamic acid and related compounds on the central nervous system. I. Central depressant effect of theanine. Chem Pharm Bull 1971;19:1257-1261.

47. LyON MR, KAPOOR MP, JUNEJA LR. The effects of L-theanine (Suntheanine $(\mathrm{R})$ ) on objective sleep quality in boys with attention deficit hyperactivity disorder (ADHD): a randomized, double-blind, placebo-controlled clinical trial. Altern Med Rev 2011;16:348-354.

48. Dodd Fl, Kennedy DO, Riby LM, Haskell-Ramsay CF. A double-blind, placebo-controlled study evaluating the effects of caffeine and L-theanine both alone and in combination on cerebral blood flow, cognition and mood. Psychopharmacology 2015;232:2563-2576.

49. Roberts KL, HaLl DA. Examining a supramodal network for conflict processing: a systematic review and novel functional magnetic resonance imaging data for related visual and auditory stroop tasks. J Cogn Neurosci 2008;20:1063-1078.

50. Godsil BP, Kiss JP, Spedding M, JAY TM. The hippocampalprefrontal pathway: the weak link in psychiatric disorders? Eur Neuropsychopharmacol 2013;23:1165-1181.

51. MARSDEN WN. Synaptic plasticity in depression: molecular, cellular and functional correlates. Prog Neuropsychopharmacol Biol Psychiatry 2013;43:168-184.

52. Zhang G, Miura Y, Yagasaki K. Effects of dietary powdered green tea and theanine on tumor growth and endogenous hyperlipidemia in hepatoma-bearing rats. Biosci Biotechnol Biochem 2002;66:711-716. 